\title{
Interference Suppression for Multiuser Downlink Transmission in Frequency-Selective Fading Channels
}

\author{
Li Guo and Yih-Fang Huang, Fellow, IEEE
}

\begin{abstract}
This paper presents an interference suppression scheme for multiuser downlink transmission in severe frequency-selective fading environments. Frequency-domain transmit beamforming and pre-equalization based on single-carrier frequency-domain equalization (SC-FDE) technique are employed to cancel co-channel interference (CCI) and to suppress intersymbol interference (ISI) with relatively low complexity. The proposed scheme effectively suppresses the ISI with a zero-forcing constraint on the CCI, and provides a viable solution to an otherwise analytically complex, if not unsolvable, problem. This paper also shows that, in frequency-selective fading channels, a multiuser system with $K$ users and $M_{t}$ base transceiver station antennas can offer lower bit error rate than a single-user system with $M_{t}-K+1$ BTS antennas, if the proposed frequency-domain multiuser beamforming is employed.
\end{abstract}

Index Terms-Co-channel interference, frequency-domain equalization, frequency-selective broadcast channels, intersymbol interference (ISI), multiantenna multiuser transmission.

\section{INTRODUCTION}

$\mathbf{T}$ HIS paper considers the problem of interference suppression for multiuser multiantenna downlink transmission in frequency-selective channels. Multiuser downlink transmission provides significant throughput improvement for wireless systems because it transmits multiple simultaneous data streams to different users [2]-[8]. However, multiuser transmission is generally subject to more interference that includes co-channel interference (CCI) due to multiple simultaneous data streams of different users, and intersymbol interference (ISI) that results from multipath fading. Therefore, interference suppression is critical to performance improvement for multiuser systems. A unique feature in downlink multiuser transmission is that the desired signal and CCI have the same spatial signature at the

Manuscript received April 12, 2007; revised March 13, 2008. First published May 23, 2007; last published August 13, 2008 (projected). The associate editor coordinating the review of this manuscript and approving it for publication was Prof. John J. Shynk. This work was completed while L. Guo was at the University of Notre Dame pursuing his Ph.D. degree. This work has been supported, in part, by the U.S. Department of the Army under Contract DAAD 16-02-C-0057-P1, and by the Indiana 21st Century Fund for Research and Technology. This work has been presented, in part, at the IEEE International Workshop on Signal Processing Advances in Wireless Communications, New York, NY, June 2005.

L. Guo is with Cisco Systems, Inc., Richardson, TX 75082 USA (e-mail: liguo2@cisco.com).

Y.-F. Huang is with the Department of Electrical Engineering, University of Notre Dame, Notre Dame, IN 46556 USA (e-mail: huang@nd.edu).

Color versions of one or more of the figures in this paper are available online at http://ieeexplore.ieee.org.

Digital Object Identifier 10.1109/TSP.2008.925958 receiver. Receive processing usually can not suppress CCI effectively in such systems. So transmit preprocessing [9] is generally preferred.

Literature abounds in proposals of and investigations on innovative schemes that explore the system throughput gain. Schubert [10] proposed a downlink beamforming scheme with an individual signal-to-interference and noise ratio (SINR) constraint. A block channel inversion (or block diagonalization) algorithm was presented in [11] to optimize power transfer to multiple antennas. Wong et al. [12] developed a multiuser solution that maximizes a lower bound for the product of SINR. In [13], a multiuser space-time block coding (STBC) scheme was proposed with an eigenmode selection approach. Multi-user schemes for frequency-selective broadcast channels were presented in [14]-[18] based on multicarrier transmission, where an ISI broadcast channel was decomposed into parallel flat channels through discrete Fourier transform (DFT). Frequency-division multiple-access (FDMA)-based schemes were studied in [14]-[18], where each subchannel is used by no more than one user, and some subchannel assignment schemes were given in [16], [17]. Space-division multiple-access (SDMA)-based multicarrier scheme was considered in [18], where all users can use any of the subchannels.

In this paper, we propose a multiuser downlink transmission scheme that effectively suppresses interference in frequency-selective fading channels. The base transceiver station (BTS) sends independent information simultaneously to multiple independent receivers through a frequency-selective broadcast channel, in which all users share the entire spectrum. Based on the idea of single-carrier frequency-domain equalization (SC-FDE) [19]-[21], the proposed scheme employs SC frequency-domain beamforming and pre-equalization at the BTS, thereby removing the CCI and suppressing the ISI. Assuming that the channel information is available at the transmitter, the optimal preweighting vectors are designed to cancel the CCI completely, namely, zero-forcing the CCI, and then to minimize the mean-squared error (MSE) for each individual user. Though this zero-forcing constraint may compromise the overall performance of interference suppression of ISI and CCI, this constraint de-couples a coupled problem in a multiuser system for which there exists no analytical solution in the literature to date. A typical approach to solve this coupled problem is to employ numerical optimization methods which are usually very complex to implement. Our constrained optimization approach thus offers a simpler and more viable solution. Furthermore, in the resulting scheme, all the complexity is born 


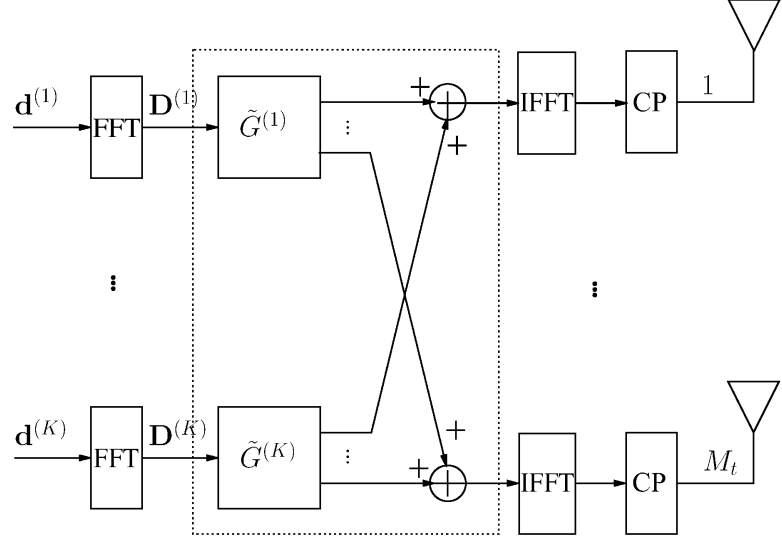

(a)

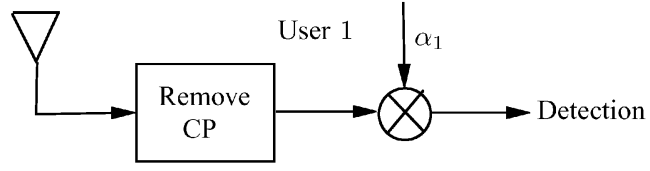

$\vdots$

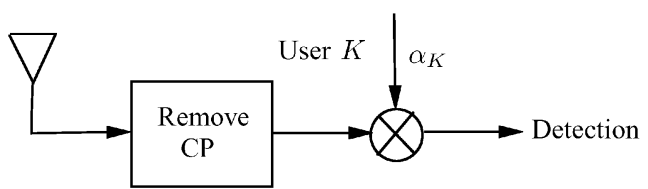

(b)

Fig. 1. Multi-user multiantenna system with frequency-domain preprocessing. (a) Transmitter and (b) receivers.

by the transmitter. The results presented in this paper show that the proposed scheme with $K$ users and $M_{t}$ BTS antennas renders lower bit error rate (BER) than a single-user system with $M_{t}-K+1$ BTS antennas.

It will be shown that the multiuser beamforming in the proposed scheme can reduce the correlation between two frequency subbands of frequency-selective spectrum, as compared to single-user beamforming. Thus, the probability of two frequency subbands being in deep fade simultaneously is smaller in a multiuser system than in a single-user system. This implies a better chance to recover the signal from the energy received from different frequency subbands in a multiuser system. Consequently, a multiuser system could provide a lower BER than a single-user scheme. This is in contrast to the result for flat-fading channels, which states that a $K$-user scheme with $M_{t}$ BTS antennas can only achieve the BER performance of a single-user scheme with $M_{t}-K+1$ antennas, see, e.g., [13].

This paper is organized as follows. In Section II, a multiuser multiantenna system model is introduced. Section III presents a frequency-domain beamforming and pre-equalization scheme as well as the derivation of the optimal preweighting parameters. In Section IV the performance of the proposed scheme is examined and compared with the single-user system. Section $\mathrm{V}$ provides simulation results that demonstrate the improved performance achieved by the proposed scheme while Section VI addresses the complexity issue. Section VII concludes the paper.

Notation: Lowercase (uppercase) letters represent time-domain (frequency-domain) quantities. Boldface denotes vectors while regular face with a tilde sign denotes matrices. Superscripts ${ }^{*},{ }^{T},{ }^{H}$ represent complex conjugate, transpose and Hermitian operations, respectively.

\section{Multiuser Multiantenna System Model}

The configuration of the proposed multiuser multiantenna system is shown in Fig. 1. Assume that there are $M_{t}$ antennas at the BTS and $K$ mobile stations (MSs) in the system. For simplicity, let there be only one receive antenna at each MS. Consider a single-carrier block-wise transmission with block length $N$. At the BTS, the data symbols (to be transmitted) to the $k$ th user are first divided into blocks of $N$ symbols denoted by $\mathbf{d}^{(k)}=\left[d_{0}^{(k)}, \ldots, d_{N-1}^{(k)}\right]^{T}$. To conduct frequency-domain transmit processing, we first pass these symbols through a serial-to-parallel converter (S/P) and an $N$-point FFT to generate the frequency-domain blocks $\mathbf{D}^{(k)}$. These frequency-domain blocks are then transformed by an $N M_{t} \times N$ preweighting matrix (combined beamforming and pre-equalization) given by

$$
\tilde{G}^{(k)}=\left[\tilde{G}_{1}^{(k)}, \tilde{G}_{2}^{(k)}, \ldots, \tilde{G}_{M_{t}}^{(k)}\right]^{T}
$$

where $\tilde{G}_{m_{t}}^{(k)}=\operatorname{diag}\left\{G_{m_{t}, 0}^{(k)}, G_{m_{t}, 1}^{(k)}, \ldots, G_{m_{t}, N-1}^{(k)}\right\}$ is an $N \times N$ diagonal matrix. After that, at each transmit branch, the signals are passed through an IFFT and each block is then transmitted along with a cyclic prefix. The cyclic prefix is inserted to eliminate the interblock interference and convert the linear convolution of the channel into a cyclic convolution. At the receiver, the cyclic prefix is first removed, then the received block $\mathbf{y}^{(k)}$ is multiplied by a scalar $\alpha_{k}$ and detection follows.

Throughout this paper, we assume a complex baseband discrete-time model with frequency-selective fading channels and additive white Gaussian noise (AWGN). Let

$$
h_{m_{t}}^{(k)}(n)=\sum_{l=0}^{L} h_{m_{t}, l}^{(k)} \delta(n-l)
$$

be the discrete-time impulse response of the $k$ th user's equivalent channel between the $m_{t}$ th BTS antenna and the receive antenna which includes the wireless propagation channel, the pulse shaping function and the receive filter impulse response. The channel coefficients $h_{m_{t}, l}^{(k)}$ are modeled as independent, zero-mean, complex Gaussian random variables with $\mathbb{E}\left[\left|h_{m_{t}, l}^{(k)}\right|^{2}\right]=\sigma_{l}^{2}$, where $\sigma_{l}^{2}$ depends on the discrete power delay profile. Normalizing the average squared gain of each link from each BTS antenna to each MS antenna, we have unity average received power, i.e., $\mathbb{E}\left[\sum_{l=0}^{L}\left|h_{m_{t}, l}^{(k)}\right|^{2}\right]=1$. Without loss of generality, we assume $\left\{h_{m_{t}, l}^{(k)}\right\}$ are uncorrelated for all $m_{t}$ and $l$, and are invariant within a data block, although they may vary from block to block. There might exist correlation between different users' channel coefficients. However, it does not affect the following derivations. 
In this multiuser system, the received block of the $k$ th user can be written as

$$
\begin{aligned}
\mathbf{y}^{(k)}=\alpha_{k} & \left(\sum_{m_{t}=1}^{M_{t}} \tilde{C}_{m_{t}}^{(k)} \tilde{F}^{H} \tilde{G}_{m_{t}}^{(k)^{H}} \tilde{F} \mathbf{d}^{(k)}\right. \\
& \left.+\sum_{m_{t}=1}^{M_{t}} \tilde{C}_{m_{t}}^{(k)} \tilde{F}^{H} \sum_{l=1, l \neq k}^{K} \tilde{G}_{m_{t}}^{(l)^{H}} \tilde{F} \mathbf{d}^{(l)}+\mathbf{v}^{(k)}\right)
\end{aligned}
$$

where $\mathbf{y}^{(k)}=\left[y_{0}^{(k)}, y_{1}^{(k)}, \ldots, y_{N-1}^{(k)}\right]^{T}$ is the received signal vector, $\mathbf{v}^{(k)}=\left[v_{0}^{(k)}, v_{1}^{(k)}, \ldots, v_{N-1}^{(k)}\right]^{T}$ is the additive Gaussian noise vector which is assumed to be zero-mean with covariance matrix $\sigma_{v}^{2} \mathbf{I}$ (where $\mathbf{I}$ denotes the identity matrix), $\tilde{C}_{m_{t}}^{(k)}$ is the $N \times$ $N$ circulant channel transfer matrix between the $m_{t}$ th BTS antenna and the $k$ th user's receive antenna, and $\tilde{F}$ is the DFT matrix to be specified below. The first column of $\tilde{C}_{m_{t}}^{(k)}$ is equal to the channel impulse response $\mathbf{h}_{m_{t}}^{(k)}=\left[h_{m_{t}, 0}^{(k)}, h_{m_{t}, 1}^{(k)}, \ldots, h_{m_{t}, L}^{(k)}\right]^{T}$ appended by $N-L$ zeros, i.e.,

$$
\tilde{C}_{m_{t}}^{(k)}=\left[\begin{array}{ccccccc}
h_{m_{t}, 0}^{(k)} & 0 & 0 & \ldots & \ldots & h_{m_{t}, 1}^{(k)} \\
h_{m_{t}, 1}^{(k)} & h_{m_{t}, 0}^{(k)} & 0 & \ldots & \ldots & \vdots \\
\vdots & h_{m_{t}, 1}^{(k)} & \ddots & \ddots & \ddots & h_{m_{t}, L}^{(k)} \\
h_{m_{t}, L}^{(k)} & \ldots & & h_{m_{t}, 0}^{(k)} & 0 & \ldots & 0 \\
0 & \ddots & & & \ddots & \vdots \\
\vdots & & & & & 0 \\
0 & \ldots & & & & 0 & h_{m_{t}, 0}^{(k)}
\end{array}\right] .
$$

The circulant matrix $\tilde{C}_{m_{t}}^{(k)}$ has an eigen-decomposition

$$
\tilde{C}_{m_{t}}^{(k)}=\tilde{F}^{H} \tilde{\Lambda}_{m_{t}}^{(k)} \tilde{F}
$$

where $\tilde{\Lambda}_{m_{t}}^{(k)}$ is a diagonal matrix whose diagonal elements are the $N$-point DFT of $\mathbf{h}_{m_{t}}^{(k)}=\left[h_{m_{t}, 0}^{(k)}, h_{m_{t}, 1}^{(k)}, \ldots, h_{m_{t}, L}^{(k)}\right]^{T}$, and the elements of the DFT matrix $\tilde{F}$ are

$$
\{\tilde{F}\}_{m, n}=\frac{1}{\sqrt{N}} e^{-i \frac{2 \pi}{N}(m-1)(n-1)} \quad 1 \leq m, n \leq N .
$$

Accordingly, for convenience and simplicity of discussion, the equivalent received frequency-domain signals are expressed as

$$
\mathbf{Y}^{(k)}=\alpha_{k}\left(\sum_{m_{t}=1}^{M_{t}} \tilde{\Lambda}_{m_{t}}^{(k)} \sum_{l=1}^{K} \tilde{G}_{m_{t}}^{(l)} \mathbf{D}^{H}+\mathbf{V}^{(k)}\right)
$$

where $\mathbf{Y}^{(k)}, \mathbf{D}^{(k)}$, and $\mathbf{V}^{(k)}$ are the DFT of $\mathbf{y}^{(k)}, \mathbf{d}^{(k)}$ and $\mathbf{v}^{(k)}$, respectively. The received signal at the $n$th bin of the $k$ th user is given by

$$
\begin{aligned}
Y_{n}^{(k)}=\alpha_{k}\left(D_{n}^{(k)} \mathbf{G}_{n}^{(k)}{ }^{H} \mathbf{H}_{n}^{(k)}\right. & \\
& \left.+\sum_{l=1, l \neq k}^{K} D_{n}^{(l)} \mathbf{G}_{n}^{(l)^{H}} \mathbf{H}_{n}^{(k)}+V_{n}^{(k)}\right)
\end{aligned}
$$

where $\mathbf{H}_{n}^{(k)}=\left[H_{1, n}^{(k)}, H_{2, n}^{(k)}, \ldots, H_{M_{t}, n}^{(k)}\right]^{T}$ with

$$
H_{m_{t}, n}^{(k)}=\sum_{l=0}^{L} h_{m_{t}, l}^{(k)} e^{-j \frac{2 \pi}{N} n l}
$$

and $\mathbf{G}_{n}^{(k)}$ is the $k$ th user's preweighting vector for the $n$th frequency bin, and its $m$ th $\left(m=1,2, \ldots, M_{t}\right)$ element is the $n$th diagonal element of $\tilde{G}_{m}^{(k)}$, i.e., $\mathbf{G}_{n}^{(k)}=$ $\left[G_{1, n}^{(k)}, G_{2, n}^{(k)}, \ldots, G_{M_{t}, n}^{(k)}\right]^{T}$ for $n=0,1, \ldots, N-1$ and $k=1,2, \ldots, K$. Then the $i$ th symbol in the received block of $k$ th user can be rewritten as

$$
\begin{aligned}
y_{i}^{(k)}= & d_{i}^{(k)}+\frac{1}{\sqrt{N}} \sum_{n=0}^{N-1}\left(\alpha_{k} \mathbf{G}_{n}^{(k)^{H}} \mathbf{H}_{n}^{(k)}-1\right) D_{n}^{(k)} e^{j 2 \pi n i / N} \\
& +\frac{\alpha_{k}}{\sqrt{N}} \sum_{n=0}^{N-1}\left(\sum_{l=1, l \neq k}^{K} D_{n}^{(l)} \mathbf{G}_{n}^{(l)^{H}} \mathbf{H}_{n}^{(k)}\right) e^{j 2 \pi n i / N} \\
& +\frac{\alpha_{k}}{\sqrt{N}} \sum_{n=0}^{N-1} V_{n}^{(k)} e^{j 2 \pi n i / N}
\end{aligned}
$$

The terms on the right-hand side of (7) are the desired signal $d_{i}^{(k)}$, the ISI due to frequency-selective multipath fading, the $\mathrm{CCI}$ from other users and additive noise. The received signal $y_{i}^{(k)}$ is clearly corrupted by both ISI and CCI. The preweighting vector $\mathbf{G}_{n}^{(k)}$, when chosen appropriately, will suppress both CCI and ISI and offer improved performance.

\section{DownLINK FREQUENCY-DomaIn BEAMFORMING AND PRE-EQUALIZATION}

Given the formulation of (7), the objective here is to cancel CCI and suppress ISI, subject to a total BTS transmit power constraint. In particular, we aim to suppress ISI under a zeroforcing constraint on CCI. The $K$-user downlink transmission is a $K$-coupled problem. The constraint of zero CCI proposed in this paper converts the coupled problems into $K$ independent problems. The resulting scheme could be suboptimal to the approach of minimizing the overall MSE for each user without the constraint. However, minimizing the overall MSE of each user generally requires numerical optimization which is usually very complex. Assume that the transmit power is equally allocated to $K$ users. Based on the signal model (3), the total transmit power of each user is

$$
P_{k}=\mathbb{E}\left[\sum_{m_{t}=1}^{M_{t}}\left\|\tilde{F}^{H} \tilde{G}_{m_{t}}^{(k)^{H}} \tilde{F} \mathbf{d}^{(k)}\right\|^{2}\right]
$$

and the CCI contributed by user $k$ to any other user $l$ is

$$
I_{k \rightarrow l}=\sum_{m_{t}=1}^{M_{t}} \tilde{C}_{m_{t}}^{(l)} \tilde{F}^{H} \tilde{G}_{m_{t}}^{(k)^{H}} \tilde{F} \mathbf{d}^{(k)}
$$

for any $k, l=1,2, \ldots, K, k \neq l$. Given a total transmit power constraint $K N \sigma_{d}^{2}$ for all users (where $\sigma_{d}^{2}$ is the average power of $d_{n}^{(k)}$ ) with known channel information, we aim to find the preweighting matrix $\tilde{G}^{(k)}$ and $\alpha_{k}$ by minimizing the MSE between the input and output for each user. The problem can be 
formulated mathematically as one of finding $\tilde{G}^{(k)}$ and $\alpha_{k}$ such that,

$$
\begin{aligned}
& \left\{\alpha_{k}, \tilde{G}^{(k)}\right\}=\arg \min _{\tilde{G}^{(k)} \in \mathbb{C}\left(N \times N M_{t}\right), \alpha_{k} \in \mathbb{C}} \mathbb{E}\left[\left\|\mathbf{y}^{(k)}-\mathbf{d}^{(k)}\right\|^{2}\right] \\
& \forall k=1,2, \ldots, K
\end{aligned}
$$

subject to

$$
\mathbb{E}\left[\sum_{m_{t}=1}^{M_{t}}\left\|\tilde{F}^{H} \tilde{G}_{m_{t}}^{(k)^{H}} \tilde{F} \mathbf{d}^{(k)}\right\|^{2}\right] \leq N \sigma_{d}^{2}, \quad \forall k=1,2, \ldots, K
$$

and

$$
\sum_{m_{t}=1}^{M_{t}} \tilde{C}_{m_{t}}^{(k)} \tilde{F}^{H} \tilde{G}_{m_{t}}^{(l) H} \tilde{F} \mathbf{d}^{(l)}=\mathbf{0} \quad 1 \leq l, k \leq K, l \neq k
$$

for all possible data vectors $\mathbf{d}^{(k)}$. Note that (9) represents the transmit power constraint for each user and (10) ensures that no users cause any interference to any other user. For simplicity of discussion, this problem is re-formulated for frequency-domain signals and parameters. The MSE of each user can also be expressed as $\mathbb{E}\left[\left\|\mathbf{Y}^{(k)}-\mathbf{D}^{(k)}\right\|^{2}\right] / N^{2}$. Thus, (8)-(10) can be reformulated as finding the vector $\mathbf{G}_{n}^{(k)}$ and $\alpha_{k}$ such that

$$
\begin{aligned}
& \left\{\mathbf{G}_{0}^{(k)}, \mathbf{G}_{1}^{(k)}, \ldots, \mathbf{G}_{N-1}^{(k)}, \alpha_{k}\right\} \\
& =\arg \min _{\mathbf{G}_{n}^{(k)} \in \mathbb{C}^{M_{t} \times 1}, 0 \leq n \leq N-1, \alpha_{k} \in \mathbb{C}} \mathbb{E}\left[\left\|\mathbf{Y}^{(k)}-\mathbf{D}^{(k)}\right\|^{2}\right] \\
& \quad \forall k=1,2, \ldots, K .
\end{aligned}
$$

subject to

$$
\sum_{n=0}^{N-1}\left\|\mathbf{G}_{n}^{(k)}\right\|^{2} \leq N, \quad 1 \leq k \leq K
$$

and

$$
\mathbf{G}_{n}^{(k){ }^{H}} \mathbf{H}_{n}^{(l)}=0, \quad 1 \leq l, k \leq K, l \neq k, 0 \leq n \leq N-1 .
$$

The optimum preweighting vector $\mathbf{G}_{n}^{(k)}$ in (11) for each bin of each user is the one that minimizes the MSE at the $k$ th MS and cancels all the CCI contributed from this user's signals to all the other users at the $n$th frequency bin.

To find the solutions for $\mathbf{G}_{n}^{(k)}$ and $\alpha_{k}$, decompose $\mathbf{G}_{n}^{(k)}$ into two parts

$$
\mathbf{G}_{n}^{(k)}=\beta_{n}^{(k)} \mathbf{W}_{n}^{(k)}
$$

where $\beta_{n}^{(k)}$ is a complex scalar and $\mathbf{W}_{n}^{(k)}$ is a unitary complex vector characterizing the direction of $\mathbf{G}_{n}^{(k)}$. One can consider $\mathbf{W}_{n}^{(k)}$ as the unitary beamforming vector for the $k$ th user's $n$th frequency band and $\beta_{n}^{(k)}$ as the pre-equalization coefficient for the $n$th frequency band of the equivalent channel after beamforming. With the constraints (12) and (13), the optimum solutions for $\mathbf{W}_{n}^{(k)}$ and $\beta_{n}^{(k)}$ must satisfy

$$
\mathbf{W}_{n}^{(k)^{H}} \mathbf{H}_{n}^{(l)}=0, \quad 1 \leq l, k \leq K, l \neq k
$$

and

$$
\sum_{n=0}^{N-1}\left|\beta_{n}^{(k)}\right|^{2} \leq N, \quad 1 \leq k \leq K
$$

\section{A. Solution of $\beta_{n}^{(k)}$ and $\alpha_{k}$}

With any beamforming vector $\mathbf{W}_{n}^{(k)}$ that satisfies (15), the received signal at the $n$th bin of the $k$ th user can be simply expressed as

$$
Y_{n}^{(k)}=\alpha_{k} D_{n}^{(k)} \beta_{n}^{(k)^{*}} \mathbf{W}_{n}^{(k)^{H}} \mathbf{H}_{n}^{(k)}+\alpha_{k} V_{n}^{(k)} .
$$

The optimum solution of $\beta_{n}^{(k)}$ and $\alpha_{k}$ for (11) as a function of $\mathbf{W}_{n}^{(k)}$ is found by minimizing the MSE of each user based on the above signal model, (17). The MSE of user $k$ is given by $\sum_{n=0}^{N-1} \epsilon_{n}^{(k)} / N^{2}$, where

$$
\epsilon_{n}^{(k)}=\mathbb{E}\left[\left|Y_{n}^{(k)}-D_{n}^{(k)}\right|^{2}\right]
$$

Furthermore, the power constraint (9) requires that $\sum_{n=0}^{N-1}\left|\beta_{n}^{(k)}\right|^{2} \leq N$ (since $\left\|\mathbf{W}_{n}^{(k)}\right\|^{2}=1$ ). Assume that time-domain symbols $\left\{d_{n}^{(k)}\right\}$ of each user form a zero-mean, independent and identically distributed (i.i.d.) data sequence with variance $\sigma_{d}^{2}$, and AWGN noise samples are statistically independent in each received block with zero-mean and variance $\sigma_{v}^{2}$. Further assume that the data symbols and AWGN noise samples are independent. Consequently, $D_{n}^{(k)}$ and $V_{n}^{(k)}$ are also independent with zero-mean and variance $N \sigma_{d}^{2}$ and $N \sigma_{v}^{2}$, respectively. Thus, the MSE at each bin is

$$
\epsilon_{n}^{(k)}=N\left(\left|1-\alpha_{k} \beta_{n}^{(k)^{*}} \mathbf{W}_{n}^{(k)^{H}} \mathbf{H}_{n}^{(k)}\right|^{2} \sigma_{d}^{2}+\left|\alpha_{k}\right|^{2} \sigma_{v}^{2}\right) .
$$

Minimizing the MSE with respect to $\alpha_{k}$ and $\beta_{n}^{(k)}$ (with constraint $\left.\sum_{n=0}^{N-1}\left|\beta_{n}^{(k)}\right|^{2} \leq N\right)$ for each user leads to the following $N+1$ equations for each user:

$$
\begin{array}{r}
\sigma_{d}^{2} \sum_{n=0}^{N-1}\left|\beta_{n}^{(k)}\right|^{2}\left|\mathbf{W}_{n}^{(k)^{H}} \mathbf{H}_{n}^{(k)}\right|^{2} \alpha_{k}+N \sigma_{v}^{2} \alpha_{k} \\
=\sigma_{d}^{2} \sum_{n=0}^{N-1} \beta_{n}^{(k)} \mathbf{W}_{n}^{(k)^{T}} \mathbf{H}_{n}^{(k)^{*}}
\end{array}
$$

and

$$
\begin{aligned}
\lambda \beta_{n}^{(k)}+\sigma_{d}^{2}\left|\alpha_{k}\right|^{2}\left|\mathbf{W}_{n}^{(k)^{H}} \mathbf{H}_{n}^{(k)}\right|^{2} \beta_{n}^{(k)} & =\alpha_{k} \mathbf{W}_{n}^{(k)^{H}} \mathbf{H}_{n}^{(k)}, \\
n & =0,1, \ldots, N-1
\end{aligned}
$$

with $\sum_{n=0}^{N-1}\left|\beta_{n}^{(k)}\right|^{2} \leq N$, where $\lambda$ is the Lagrange multiplier. The solutions of $\alpha_{k}$ and $\beta_{n}^{(k)}$ from the above equations are, respectively,

$$
\alpha_{k, o}=\sqrt{\frac{1}{N} \sum_{n=0}^{N-1} \frac{\left|\mathbf{W}_{n}^{(k){ }^{H}} \mathbf{H}_{n}^{(k)}\right|^{2}}{\left(\left|\mathbf{W}_{n}^{(k)^{H}} \mathbf{H}_{n}^{(k)}\right|^{2}+\sigma_{v}^{2} / \sigma_{d}^{2}\right)^{2}}}
$$


and

$$
\beta_{n, o}^{(k)}=\frac{1}{\alpha_{k}} \frac{\mathbf{W}_{n}^{(k)^{H}} \mathbf{H}_{n}^{(k)}}{\left|\mathbf{W}_{n}^{(k)^{H}} \mathbf{H}_{n}^{(k)}\right|^{2}+\sigma_{v}^{2} / \sigma_{d}^{2}} .
$$

\section{B. Optimum Solution of $\mathbf{W}_{n}^{(k)}$}

To obtain the optimum solution of $\mathbf{W}_{n}^{(k)}$ which minimizes the MSE for each and satisfies (15), rewrite the MSE for the $k$ th user based on the expressions for the optimum solutions of $\alpha_{k}$ and $\beta_{n}^{(k)}$ as a function of $\mathbf{W}_{n}^{(k)}$ in (22) and (23):

$$
\begin{aligned}
\mathrm{MSE}_{k}= & \frac{\sigma_{d}^{2}}{N} \sum_{n=0}^{N-1}\left|1-\frac{\left|\mathbf{W}_{n}^{(k)^{H}} \mathbf{H}_{n}^{(k)}\right|^{2}}{\left|\mathbf{W}_{n}^{(k)^{H}} \mathbf{H}_{n}^{(k)}\right|^{2}+\sigma_{v}^{2} / \sigma_{d}^{2}}\right|^{2} \\
& +\frac{\sigma_{v}^{2}}{N} \sum_{n=0}^{N-1} \frac{\left|\mathbf{W}_{n}^{(k)^{H}} \mathbf{H}_{n}^{(k)}\right|^{2}}{\left(\left|\mathbf{W}_{n}^{(k)^{H}} \mathbf{H}_{n}^{(k)}\right|^{2}+\sigma_{v}^{2} / \sigma_{d}^{2}\right)^{2}} \\
= & \frac{1}{N} \sum_{n=0}^{N-1} \frac{\sigma_{d}^{2}}{\left|\mathbf{W}_{n}^{(k)^{H}} \mathbf{H}_{n}^{(k)}\right|^{2} \sigma_{d}^{2} / \sigma_{v}^{2}+1} .
\end{aligned}
$$

Minimizing $\mathrm{MSE}_{k}$ is equivalent to maximizing $\left|\mathbf{W}_{n}^{(k)^{H}} \mathbf{H}_{n}^{(k)}\right|^{2}$ [subject to $\left\|\mathbf{W}_{n}^{(k)}\right\|^{2}=1$ and constraint (15)]. The solution of $\mathbf{W}_{n}^{(k)}$ is the orthonormal projection of $\mathbf{H}_{n}^{(k)}$ onto the null space of matrix

$$
\tilde{H}_{n}^{(k)} \triangleq\left(\mathbf{H}_{n}^{(1)}, \ldots, \mathbf{H}_{n}^{(k-1)}, \mathbf{H}_{n}^{(k+1)}, \ldots, \mathbf{H}_{n}^{(K)}\right)^{H}
$$

and is given by

$$
\mathbf{W}_{n, o}^{(k)}=\frac{\left(\tilde{I}-\tilde{H}_{n}^{(k)^{H}}\left(\tilde{H}_{n}^{(k)} \tilde{H}_{n}^{(k)^{H}}\right)^{-1} \tilde{H}_{n}^{(k)}\right) \mathbf{H}_{n}^{(k)}}{\sqrt{\mathbf{H}_{n}^{(k)^{H}}\left(\tilde{I}-\tilde{H}_{n}^{(k)^{H}}\left(\tilde{H}_{n}^{(k)} \tilde{H}_{n}^{(k)^{H}}\right)^{-1} \tilde{H}_{n}^{(k)}\right) \mathbf{H}_{n}^{(k)}}} .
$$

The beamforming vector for user $k$ cancels the interference from user $k$ to all the other users. From the solution in (26), we should note that the system configuration $M_{t} \geq K$ is a necessary condition for the existence of $\mathbf{W}_{n, o}^{(k)}$.

In this proposed multiuser system, the downlink multiuser ISI channel is decomposed into $K$ parallel single-user ISI channels. For each user, the CCI from other users' data stream is completely removed. We can think of $\mathbf{W}_{n}^{(k)^{H}} \mathbf{H}_{n}^{(k)}$ as the effective channel at the $n$th frequency bin of the $k$ th user and $\beta_{n}^{(k)}$ as the frequency-domain pre-equalization parameter for each bin of each user. The beamforming vector $\mathbf{W}_{n}^{(k)}$ is chosen to maximize the SNR of the effective channel at each bin of each user, which is $\left|\mathbf{W}_{n}^{(k)^{H}} \mathbf{H}_{n}^{(k)}\right|^{2} \sigma_{d}^{2} / \sigma_{v}^{2}$. And $\beta_{n}^{(k)}$ and $\alpha_{k}$ are chosen to minimize the MSE of each user at the receiver for the effective channel with a power constraint. the SNR of the effective channel, $\left|\mathbf{W}_{n}^{(k)^{H}} \mathbf{H}_{n}^{(k)}\right|^{2} \sigma_{d}^{2} / \sigma_{v}^{2}$, also depends on the spatial correlation between different users through $\left|\mathbf{W}_{n}^{(k)^{H}} \mathbf{H}_{n}^{(k)}\right|$. The spa- tial correlation is measured by normalized inner product of two users' channel vector $\left(\mathbf{H}_{n}^{\left(k_{1}\right)}\right.$ and $\left.\mathbf{H}_{n}^{\left(k_{2}\right)}\right)$. Smaller spatial correlation results in larger $\left|\mathbf{W}_{n}^{(k)^{H}} \mathbf{H}_{n}^{(k)}\right|$ while larger spatial correlation yields smaller $\left|\mathbf{W}_{n}^{(k)^{H}} \mathbf{H}_{n}^{(k)}\right|$. We can explore this feature to further improve the system performance through some user scheduling scheme. One scheme is to divide all the users into several subsets such that the spatial correlation within each subset is smaller than some threshold. At each time slot, the BTS only schedules the transmission to users within one subset. In this way, we can further reduce the signal strength loss due to CCI cancellation and better performance can be addressed.

\section{Performance Analysis}

In this section, we compare the performance of the proposed multiuser system to that of a single-user system [22] for frequency-selective channels. Both systems employ single-carrier transmission and the energy of each symbol is spread over the entire spectrum. In frequency-selective environments, it is quite possible that some parts of the spectrum are in deep fade while others are not. Consequently, even when some subbands of the channel are in deep fade, one can still recover the symbols from other subbands. Smaller correlation between different subbands would imply a greater chance for such recoveries. Thus, better BER performance can be expected.

To further illustrate this, we compare a two-user system with $M_{t}+1$ transmit antennas to a single-user system with $M_{t}$ BTS antennas. Through the beamforming vector $\mathbf{W}_{n}^{(k)}$, a two-user broadcast channel is decomposed into two equivalent parallel single-user channels with $M_{t}$ BTS antennas. In this section, we will show that the correlation between any two subbands of the equivalent channel (after beamforming $\mathbf{W}_{n}^{(k)}$ ) in a two-user system is smaller than that in a single-user system. Hence, a two-user system may render better BER performance.

\section{A. Single-User System}

In a single-user system [22] with $M_{t}$ BTS antennas and one MS antenna, the unitary transmit beamforming vector for the $n$th frequency bin is $\mathbf{H}_{n} /\left\|\mathbf{H}_{n}\right\|$. With transmit beamforming, the equivalent channel is $\left\|\mathbf{H}_{n}\right\|$, and the power of the equivalent channel is

$$
A_{n}=\left\|\mathbf{H}_{n}\right\|^{2}=\sum_{p=1}^{M_{t}}\left|H_{p, n}\right|^{2}
$$

where $H_{p, n}=\sum_{l=0}^{L} h_{p, l} e^{-j 2 \pi n l / N}$. As assumed in Section II, the time-domain channel coefficients $h_{p, l}$ are independent complex Gaussian random variable with zero mean and variance $\sigma_{l}^{2}$, and $\sum_{l=0}^{L} \sigma_{l}^{2}=1$. So $H_{p, n}$ is also a complex Gaussian random variables with zero mean and unity variance. The mean and variance of $A_{n}$ are, respectively,

$$
\begin{aligned}
\mu_{A_{n}} & =\mathbb{E}\left[A_{n}\right]=M_{t} \\
\sigma_{A_{n}}^{2} & =\mathbb{E}\left[\left(A_{n}-\mu_{A_{n}}\right)^{2}\right]=M_{t} .
\end{aligned}
$$


The correlation coefficient between $A_{n}$ and $A_{m}$ is

$$
\begin{aligned}
\rho_{A_{n}, A_{m}} & \triangleq \frac{\mathbb{E}\left[\left(A_{n}-\mu_{A_{n}}\right)\left(A_{m}-\mu_{A_{m}}\right)\right]}{\sqrt{\mathbb{E}\left[\left(A_{n}-\mu_{A_{n}}\right)^{2}\right] \mathbb{E}\left[\left(A_{n}-\mu_{A_{n}}\right)^{2}\right]}} \\
& =\frac{\mathbb{E}\left[A_{n} A_{m}\right]-M_{t}^{2}}{M_{t}} \\
& =\rho_{1}^{2}(n-m)+\rho_{2}^{2}(n-m)
\end{aligned}
$$

where

$$
\begin{aligned}
& \rho_{1}(n-m)=\sum_{l=0}^{L} \sigma_{l}^{2} \cos \left(\frac{2 \pi(n-m) l}{N}\right) \\
& \rho_{2}(n-m)=\sum_{l=0}^{L} \sigma_{l}^{2} \sin \left(\frac{2 \pi(n-m) l}{N}\right) .
\end{aligned}
$$

For detailed derivation, please see Appendix I. The correlation coefficient $\rho\left(A_{n}, A_{m}\right)$ does not depend on the number of antennas $M_{t}$.

\section{B. Multiuser System}

We consider a two-user system with $M_{t}+1$ BTS antennas. The unitary beamforming vector for user 1 is

$$
\mathbf{W}_{n}^{(1)}=\frac{\left(\mathbf{I}-\frac{\mathbf{H}_{n}^{(2)} \mathbf{H}_{n}^{(2)}{ }^{H}}{\left\|\mathbf{H}_{n}^{(2)}\right\|^{2}}\right) \mathbf{H}_{n}^{(1)}}{\sqrt{\left\|\mathbf{H}_{n}^{(1)}\right\|^{2}-\frac{\mathbf{H}_{n}^{(1)}{ }^{H} \mathbf{H}_{n}^{(2)} \mathbf{H}_{n}^{(2)}{ }^{H} \mathbf{H}_{n}^{(1)}}{\left\|\mathbf{H}_{n}^{(2)}\right\|^{2}}}} .
$$

With transmit beamforming, the equivalent channel of user 1 is $\mathbf{W}_{n}^{(1)^{H}} \mathbf{H}_{n}^{(1)}$, and the power of the equivalent channel is

$$
B_{n}=\left\|\mathbf{H}_{n}^{(1)}\right\|^{2}-\frac{\mathbf{H}_{n}^{(1)^{H}} \mathbf{H}_{n}^{(2)} \mathbf{H}_{n}^{(2)^{H}} \mathbf{H}_{n}^{(1)}}{\left\|\mathbf{H}_{n}^{(2)}\right\|^{2}} .
$$

Given $\mathbf{H}_{n}^{(2)}$, the conditional mean and variance of $B_{n}$ are (see Appendix II for detailed derivation)

$$
\begin{aligned}
\mu_{B_{n}}\left(\mathbf{H}_{n}^{(2)}\right) & =\mathbb{E}\left[\left\|\mathbf{H}_{n}^{(1)}\right\|^{2}\right]-\frac{\mathbf{H}_{n}^{(2)^{H}} \mathbb{E}\left[\mathbf{H}_{n}^{(1)} \mathbf{H}_{n}^{(1)^{H}}\right] \mathbf{H}_{n}^{(2)}}{\left\|\mathbf{H}_{n}^{(2)}\right\|^{2}} \\
& =M_{t}+1-1=M_{t}
\end{aligned}
$$

and

$$
\sigma_{B_{n}}^{2}\left(\mathbf{H}_{n}^{(2)}\right)=M_{t} .
$$

Thus the mean and variance of $B_{n}$ are $\mu_{B_{n}}=M_{t}$ and $\sigma_{B_{n}}^{2}=$ $M_{t}$, respectively.

Given $\mathbf{H}_{n}^{(2)}$ and $\mathbf{H}_{m}^{(2)}$, the correlation between $B_{n}$ and $B_{m}$ is

$$
\begin{aligned}
\mathbb{E}\left[B_{n} B_{m} / \mathbf{H}_{n}^{(2)},\right. & \left.\mathbf{H}_{m}^{(2)}\right] M_{t}^{2}+\left(\rho_{1}^{2}(n-m)+\rho_{2}^{2}(n-m)\right) \\
& \times\left(M_{t}-1+\frac{\left|\mathbf{H}_{n}^{(2)^{H}} \mathbf{H}_{m}^{(2)}\right|^{2}}{\left\|\mathbf{H}_{n}^{(2)}\right\|^{2}\left\|\mathbf{H}_{m}^{(2)}\right\|^{2}}\right) .
\end{aligned}
$$

We know that $\left|\mathbf{H}_{n}^{(2)^{H}} \mathbf{H}_{m}^{(2)}\right|^{2} \leq\left\|\mathbf{H}_{n}^{(2)}\right\|^{2}\left\|\mathbf{H}_{m}^{(2)}\right\|^{2}$. So the correlation satisfies $\mathbb{E}\left[B_{n} B_{m} / \mathbf{H}_{n}^{(2)}, \mathbf{H}_{m}^{(2)}\right] \leq M_{t}^{2}+M_{t}\left(\rho_{1}^{2}(n-\right.$ $\left.m)+\rho_{2}^{2}(n-m)\right)$. The equalities are achieved if and only if $\mathbf{H}_{n}^{(2)}=a \mathbf{H}_{m}^{(2)}$ ( $a$ is a complex scalar). Therefore, the correlation of $B_{n}$ and $B_{m}$ is

$$
\begin{aligned}
\mathbb{E}\left[B_{n} B_{m}\right] & =\mathbb{E}_{\mathbf{H}_{n}^{(2)}, \mathbf{H}_{m}^{(2)}}\left[\mathbb{E}\left[B_{n} B_{m} / \mathbf{H}_{n}^{(2)}, \mathbf{H}_{m}^{(2)}\right]\right] \\
& \leq M_{t}^{2}+M_{t}\left(\rho_{1}^{2}(n-m)+\rho_{2}^{2}(n-m)\right) .
\end{aligned}
$$

The correlation coefficient of $B_{n}$ and $B_{m}$ is

$$
\begin{aligned}
\rho_{B_{n}, B_{m}} & =\frac{\mathbb{E}\left[B_{n} B_{m}\right]-\mu_{B_{n}} \mu_{B_{m}}}{\sqrt{\sigma_{B_{n}}^{2} \sigma_{B_{m}}^{2}}} \\
& \leq \rho_{1}^{2}(n-m)+\rho_{2}^{2}(n-m) .
\end{aligned}
$$

From all the results above, we can conclude

$$
\rho_{B_{n}, B_{m}}<\rho_{A_{n}, A_{m}} .
$$

The inequality (39) implies that the probability of both frequency bins $n$ and $m$ being in deep fade is smaller in a two-user system than in a single-user system. Hence, it is conceivable that a two-user system can achieve a lower BER than a single-user system can. This will be verified through simulation results in the following section. Furthermore, increasing the number of users would reduce the correlation coefficient $\rho_{B_{n}, B_{m}}$, thereby improving the BER performance further, as to be shown in the next section.

\section{Channel Estimation and Imperfect Channel Information}

The above discussions assume perfect channel state information (CSI) $\mathbf{h}_{m}^{(k)}$ at the BTS, though that is not always true in practice. In this subsection, we consider channel estimation in multiuser systems and the impact of imperfect CSI on the performance of multiuser systems.

Channel reciprocity usually exists between the downlink and uplink channels in time-division duplex (TDD) systems. Exploring this property, we can use the uplink transmission to estimate CSI for downlink transmission in TDD systems. The BTS schedules some pilot slots on the uplink, and during each pilot slot it schedules some of the users to transmit pilot sequences. One choice of pilot sequence is a set of orthogonal sequences generated from Golay complementary sequences [23], [24]. Those orthogonal sequences have excellent autocorrelation characteristics and low aperiodic cross correlation [23], [24]. Assume $N_{p}$ orthogonal sequences generated from Golay complementary sequences and denote them as $\mathcal{C}_{p}=\left\{\vec{c}_{1}, \vec{c}_{2}, \ldots, \vec{c}_{N_{p}}\right\}$. The BTS schedules $N_{p}$ users to transmit a pilot block during each uplink pilot slot. Different users transmit different $\vec{c}_{n}$. The received pilot signal at the $m_{t}$ th BTS antenna is

$$
\vec{y}_{p}=\sum_{n=1}^{N_{p}} \sum_{l=0}^{L} h_{m_{t}, l}^{(n)} \vec{c}_{n}(t-l)+\vec{v} .
$$


We estimate the channel taps by calculating the correlation between $\vec{y}_{p}$ and each $\vec{c}_{n}(t-l)$. We first take $\vec{c}_{1}$ and calculate the correlation between $\vec{y}_{p}$ and $\vec{c}_{1}$. The result is $h_{m_{t}, 0}^{(1)}+\hat{v}$, where $\hat{v}$ is due to the correlation between $\vec{c}_{1}$ and $\vec{c}_{n}(t-l)$ $(n \neq 1, l=0,1, \ldots L)$, the correlation between $\vec{c}_{1}$ and $\vec{c}_{1}(t-l)$ $(l=1,2, \ldots L)$ and noise. Accordingly, the estimate for $h_{m_{t}, 0}^{(1)}$ is $\hat{h}_{m_{t}, 0}^{(1)}=h_{m_{t}, 0}^{(1)}+\hat{v}$. After we have obtained $\hat{h}_{m_{t}, 0}^{(1)}$, we remove the contribution of $\vec{c}_{1}$ by $\vec{y}_{p}=\vec{y}_{p}-\hat{h}_{m_{t}, 0}^{(1)} \vec{c}_{1}$. We then estimate $\hat{h}_{m_{t}, 1}^{(1)}$ by calculating the correlation between the new $\vec{y}_{p}$ and $\vec{c}_{1}(t-1)$. We repeat the above calculation with updated $\vec{y}_{p}$ and $\vec{c}_{1}(t-l)$ to estimate other taps $h_{m_{t}, l}^{(1)}(l=2,3, \ldots, L)$. Then we repeat the above procedure for each $\vec{c}_{n}$ to estimate the channel taps for all the users transmitting during the current pilot slot.

In a system where the reciprocity between the downlink and uplink channels does not exist [e.g., frequency division duplex (FDD)], the uplink channel information can not be used directly for downlink transmission. One approach to estimate the CSI is for the BTS to transmit pilots during some downlink pilot slot. Users estimate the downlink channel based on the received pilot signals and then feedback to BTS. We can still use the method described above to estimate the channel taps by employing the orthogonal sequences. We generate $M_{t}$ orthogonal sequences $\left\{\vec{c}_{1}, \vec{c}_{2}, \ldots, \vec{c}_{M_{t}}\right\}$. The BTS schedules some pilot slot and transmits different $\vec{c}_{m_{t}}$ from different BTS antenna. The received pilot signal at user $k$ is

$$
\vec{y}_{k}=\sum_{m_{t}=1}^{M_{t}} \sum_{l=0}^{L} h_{m_{t}, l}^{(k)} \vec{c}_{m_{t}}(n-l)+\vec{v}_{k} .
$$

Then at each user, we estimate the channel taps by calculating the correlation between $\vec{y}_{k}$ and $\vec{c}_{m_{t}}(t-l)(l=0,1, \ldots L)$ and following the same procedure described in the above paragraph.

Naturally, performance degradation can occur due to imperfect CSI in both multiuser and single-user systems. In a single-user system with minimum MSE (MMSE) pre-equalization [22], imperfect $\hat{\mathbf{h}}_{m}$ results in an imperfect pre-equalization parameter $\hat{\mathbf{G}}_{n}$. Then the received useful signal power would be reduced and the error contained in the received signal could increase. As a result, the performance of ISI suppression is impaired. In a multiuser system with imperfect CSI, a beamforming vector $\hat{\mathrm{g}}_{n}^{(k)}$ is chosen such that it is orthogonal to the estimated channel matrix $\hat{\tilde{H}}_{n}^{(k)}$ and the inner product $\left|\hat{\mathbf{g}}_{n}^{(k)^{H}} \hat{\mathbf{H}}_{n}^{(k)}\right|$ is maximized. Then imperfect pre-equalization coefficients $\hat{\beta}^{(k)}$ and $\hat{\alpha}_{k}$ are obtained accordingly. Thus, the received signal at the $k$ th MS with imperfect CSI is given by

$$
\begin{aligned}
Y_{n}^{(k)}=\hat{\alpha_{k}} & \left(\hat{\beta}_{n}^{(k)^{*}} \hat{\mathbf{g}}_{n}^{(k)^{H}} \mathbf{H}_{n}^{(k)} D_{n}^{(k)}\right. \\
& \left.\quad+\sum_{l=1, l \neq k}^{K} \hat{\beta}_{n}^{(l)^{*}} \hat{\mathrm{g}}_{n}^{(l)^{H}} \mathbf{H}_{n}^{(k)} D_{n}^{(l)}+V_{n}^{(k)}\right) .
\end{aligned}
$$

One can see that the imperfect CSI in a multiuser system can impact the received signal in a number of ways. First, the CCI term in the received signal becomes nonzero. Second, the ISI residual in the received signal is increased. Third, the useful signal power is reduced. All these factors contribute to a reduction in the signal-to-error ratio at each MS and the performance is thus compromised. A BER floor could also occur.

Compared with a single-user system, a multiuser system is generally expected to suffer more from the imperfect channel information, because of the impairment to both CCI suppression and ISI suppression. Intuitively, each user in a multiuser system can still enjoy more frequency diversity than in a single-user system when the CSI is not known perfectly, and when the error in the channel estimate is not large. However, as the CSI error increases, more $\mathrm{CCI}$ arises in a multiuser system and thus the performance deteriorates more in a multiuser system than in a single-user system. Some quantitative analysis is given in Section VI where simulation results are presented.

\section{COMPLEXITY ISSUES}

In this paper, the number of complex multiplications is used as a measure of computational complexity. There are two major parts of this complexity: the complexity of calculating parameters $\mathbf{G}_{n}^{(k)}$ and $\alpha_{k}$ for each user and that of the processing to obtain each symbol output in $\mathbf{y}^{(k)}$ for each user.

The computational complexity of transmitting one block ( $N$ symbols) for each user is in the order of $M_{t} \log _{2} N$ per symbol, which is mainly born by the BTS. At the MS, the only required computation is one scalar multiplication with $\alpha_{k}$. At the BTS, the computation needed to transmit $\left\{\mathbf{d}^{(1)}, \ldots, \mathbf{d}^{(K)}\right\}$ to the $K$ users includes the following:

- $K$ FFTs: $K N / 2 \log _{2} N$ complex multiplications;

- $M_{t}$ FFTs: $M_{t} N / 2 \log _{2} N$ complex multiplications;

- Operation with $\tilde{G}^{(k)}$ for each user: $K N M_{t}$ complex multiplications.

Thus, the processing complexity is $\left(1 / 2+M_{t} /(2 K)\right) \log _{2} N+$ $M_{t}+1$ complex multiplications per symbol and per user.

The most significant complexity in calculating the parameters is that of calculating $\mathbf{W}_{n}^{(k)}$ for each frequency bin of each user. To obtain $\mathbf{W}_{n}^{(k)}$ in (26), one needs to compute the inverse of $\tilde{H}_{n}^{(k)} \tilde{H}_{n}^{(k)^{H}}$ in (26) which has a complexity in the order of $\mathcal{O}\left((K-1)^{3}\right)$, for $\tilde{H}_{n}^{(k)} \tilde{H}_{n}^{(k)^{H}}$ is a $(K-1) \times(K-1)$ matrix. Furthermore, we need approximately $(K(K-1) / 2) M_{t}$ complex multiplications to calculate $\tilde{H}_{n}^{(k)} \tilde{H}_{n}^{(k)^{H}}$ and $M_{t}^{2} K+$ $M_{t}(K-1)^{2}$ complex multiplications for the calculation of the other product in (26). So the computational complexity needed to obtain all the beamforming and pre-equalization parameters is in the order of $N\left(M_{t}^{2} K+M_{t}(K-1)(3 K-2) / 2+\mathcal{O}((K-\right.$ $\left.\left.1)^{3}\right)\right)$ ) complex multiplications per user. This complexity is similar to that of a multicarrier multiuser system [18].

We also need to consider the complexity used in channel estimation. Assume the length of orthogonal sequences is $L_{p}$. In a TDD system, we need to calculate the cross-correlation between $\vec{y}_{p}$ and $\vec{c}_{n}$ with different delay shifts. Each cross-correlation needs roughly $L_{p}$ complex multiplications. So the total number of complex multiplications involved in the channel estimation 
for each user is in the order of $\mathcal{O}\left(L_{p} M_{t}(L+1)\right)$. Similarly, in a non-TDD system, we also need to calculate the cross-correlation between $\vec{y}_{k}$ and orthogonal sequences. The complexity of each user is in the order of $\mathcal{O}\left(L_{p} M_{t}(L+1)\right)$ complex multiplications.

\section{Simulation RESUlts}

\section{A. Configuration}

To examine the scheme proposed in Section III, we conducted simulation experiments that employed the quadrature-phase-shift-keying (QPSK) modulation for BER performance evaluation. The size of transmission block (which is the size of FFT and IFFT), $N$, was varied in accordance with channel models. For each simulation, 1,000 data blocks, each of which consisting of $N$ symbols were transmitted over more than 10,000 independent sets of channel realizations.

The results for both the single-user system [22] and the multicarrier multiuser system [18] are shown and compared. In the single-user system, there are multiple BTS antennas and one antenna at the receiver. The linear minimum mean-squared error (LMMSE) criterion is used to obtain the transmit pre-equalization matrix by minimizing the received MSE at the receiver [22]. In the multicarrier system, a multiuser OFDM scheme is employed. It is assumed that there are $M_{t}$ BTS antennas and one MS antenna in the OFDM-based system. Two transmit schemes are considered here. The first is FDMA, where each user uses one set of subbands and each tone is only occupied by one user. The subchannel allocation is done by maximizing the sum of all subbands' SNR, subject to a given number of subbands used by each user, namely, the data-rate requirement of each user. Another transmit scheme is SDMA, where each user uses all the subbands and the $\mathrm{CCI}$ is canceled by transmit preweighting vector which is orthogonal to all other user's channels at each subchannel. Since each user only uses a subset of subbands in the FDMA scheme, it requires a larger modulation constellation than the SDMA scheme to support the same data rate. It has been shown in [18] that the SDMA scheme provides better BER performance than the FDMA scheme does in supporting the same data rate. As such, we only compare the proposed system with multicarrier system employing the SDMA scheme.

\section{B. Channel Model}

The frequency-selective channels used in this section are based on the following delay profiles.

1) Multiray Uniform Power Profile: The multiray power profile has $L(\geq 1)$ rays with uniform power $1 / L$. The rays are uniformly spaced by the symbol interval and are modeled as independent complex Gaussian random variables. This uniform power profile can be used to simulate the worst kind of ISI channels. In particular, a six-ray profile was employed in the simulation, which is a standard profile used in GSM systems [25]. The root-mean-square (rms) delay spread is $1.408 T_{s}$ where $T_{s}$ is the symbol duration. For the 6-ray uniform power channel profile, the size of FFT and IFFT (i.e., the size of transmission

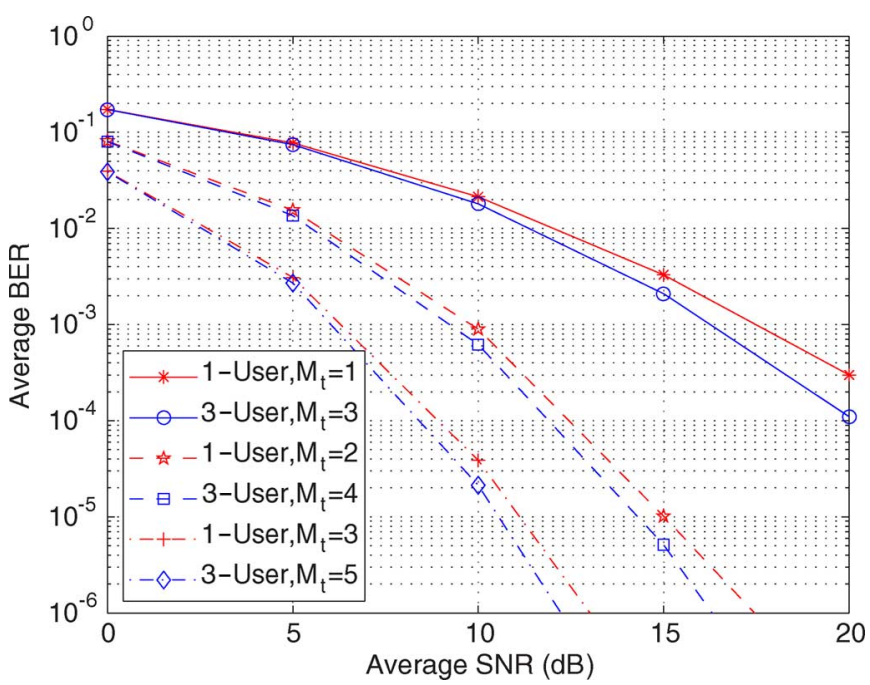

Fig. 2. Performance comparison between a three-user scheme and a single-user scheme (SUI-5 power profile).

data block $\mathbf{d}^{(k)}$ ) was chosen to be 64 in our simulation for reasonable transmission efficiency.

2) SUI-5 Channel Profile: The SUI-5 model is one of the six channel models adopted by IEEE 802.16 for evaluating broadband wireless systems in the $2-11 \mathrm{GHz}$ bands. "SUI- 5 " is a high delay model associated with the use of omnidirectional antennas in suburban hilly environments. The channel has a maximum delay spread of $10 \mu \mathrm{s}$, and an rms delay spread of $3.05 \mu \mathrm{s}$. It has three echoes, at 0,4 , and $10 \mu \mathrm{s}$, modeled as independent complex Gaussian random variables with the relative power of $0,-5,-10 \mathrm{~dB}$, respectively. For the SUI- 5 channel model, the size of FFT and IFFT was chosen to be 128 .

The following Sections, VI-C and VI-D, will present simulation results of average BER to compare the proposed multiuser system with the single-user system and multicarrier multiuser SDMA scheme. The impact of multiuser beamforming on the correlation between subbands is further investigated in Section VI-E and it is shown that multiuser beamforming with a larger number of users can provide better BER performance. The impact of imperfect channel information is studied in Section VI-F.

\section{Comparison With Single-User System}

In Figs. 2 and 3, the average BER results for various channel models are shown to compare the performance of the proposed multiuser system with that of a single-user system. The comparison is based on the same multiantenna diversity order for both systems, i.e., a multiuser system with $K$ users and $M_{t}$ transmit antennas is compared to a single-user system with $M_{t}-K+1$ transmit antennas. Both systems have the same multiantenna diversity order $M_{t}-K+1$ [26]. Fig. 2 shows the BER performance of a three-user system with an SUI-5 power profile, comparing with that of a single-user system. The number of BTS antennas in the three-user system $(K=3)$ varies from three to five (i.e., $M_{t}=3,4,5$ ), compared with one to three BTS antennas (i.e., $M_{t}-K+1=1,2,3$ ) in the single-user system. A close 


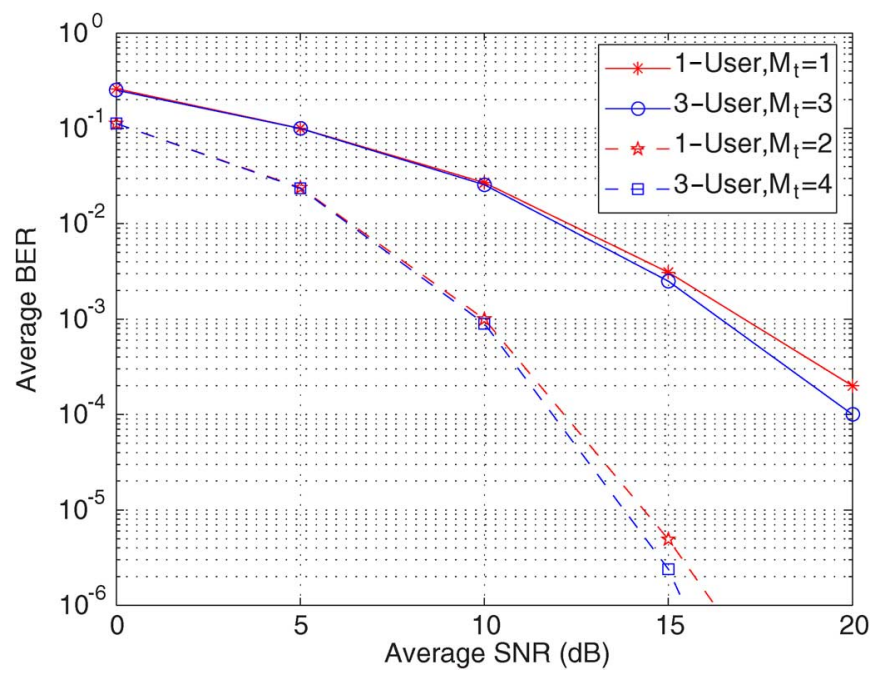

Fig. 3. Performance comparison between a three-user scheme and a single-user scheme (six-ray uniform power profile).

examination of Fig. 2 reveals that the multiuser beamforming provides better BER performance than the single-user scheme in frequency-selective fading systems. These simulation results are consistent with the analytical results derived in Section III. Similar results are observed in Fig. 3, where six-ray uniform power model was employed.

\section{Performance Comparison With Multicarrier System}

We now compare the BER performance of the proposed multiuser system with that of an uncoded OFDM-based multicarrier multiuser system employing SDMA scheme. The results for a three-user system in six-ray uniform power profile and in SUI-5 model are shown in Figs. 4 and 5, respectively. We can see that the proposed SC multiuser system significantly outperforms the OFDM-based system. There are two reasons. First, the OFDM-based multicarrier system does not explore any frequency diversity, while the single-carrier system does. Second, the multiuser scheme proposed in this paper can achieve more frequency diversity (than single-user single-carrier system) due to multiuser beamforming. It is important to note that the proposed scheme's computational complexity is comparable to that of the OFDM-based system, yet offers better BER performance.

\section{E. Correlation Between Different Subbands}

To further study the correlation issue, we looked into the BER performance of multiuser systems with different configurations. In Fig. 6, the BER performance is compared for a three-user system with three BTS antennas, a four-user system with four BTS antennas and a seven-user system with seven BTS antennas. These configurations have the same multiantenna diversity order of 1. It is observed that the system with seven users has the best BER performance. This suggests that multiuser transmit beamforming with more users results in smaller correlation between different subbands.

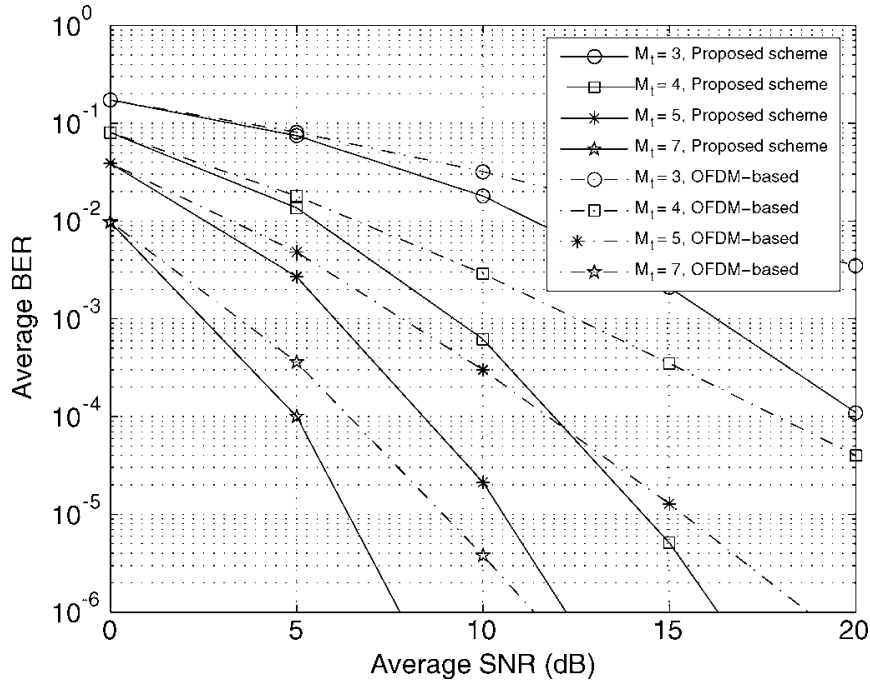

Fig. 4. BER performance comparison between a single-carrier three-user scheme and an OFDM-based three-user scheme (SUI-5 power profile).

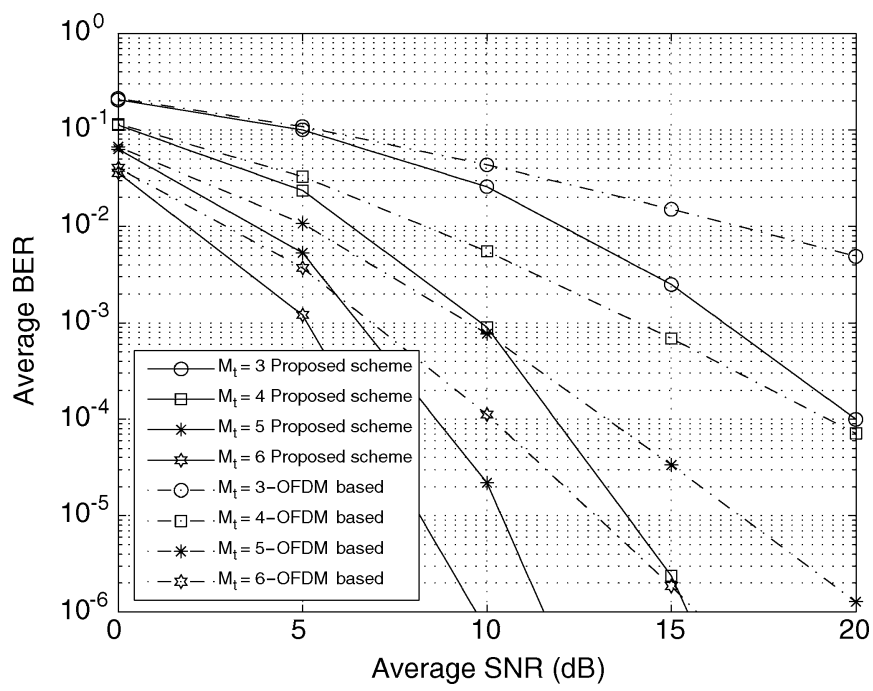

Fig. 5. BER performance comparison between a single-carrier three-user scheme and an OFDM-based three-user scheme (six-ray uniform power profile).

\section{F. Impact of Imperfect CSI}

Fig. 7 examines the BER performance with imperfect channel information, where a three-user system with three BTS antennas is compared with a single-user system with one BTS antenna. These two systems have the same multiantenna diversity order, i.e., 1 . The SUI-5 channel model was again employed. It is seen that the BER performance of both multiuser system and single-user system is impaired by the imperfect CSI and it deteriorates as the channel estimation error increases. At CSI MSE $=-30 \mathrm{~dB}$, the multiuser system still has better BER than the single-user system, and it even outperforms the single-user system with perfect CSI. However, at CSI MSE $=-20 \mathrm{~dB}$, the single-user system outperforms the multiuser system due to significant $\mathrm{CCI}$ residual existing in the multiuser system. Similar results can be observed in Fig. 8, where the BER performance of a three-user system with four 


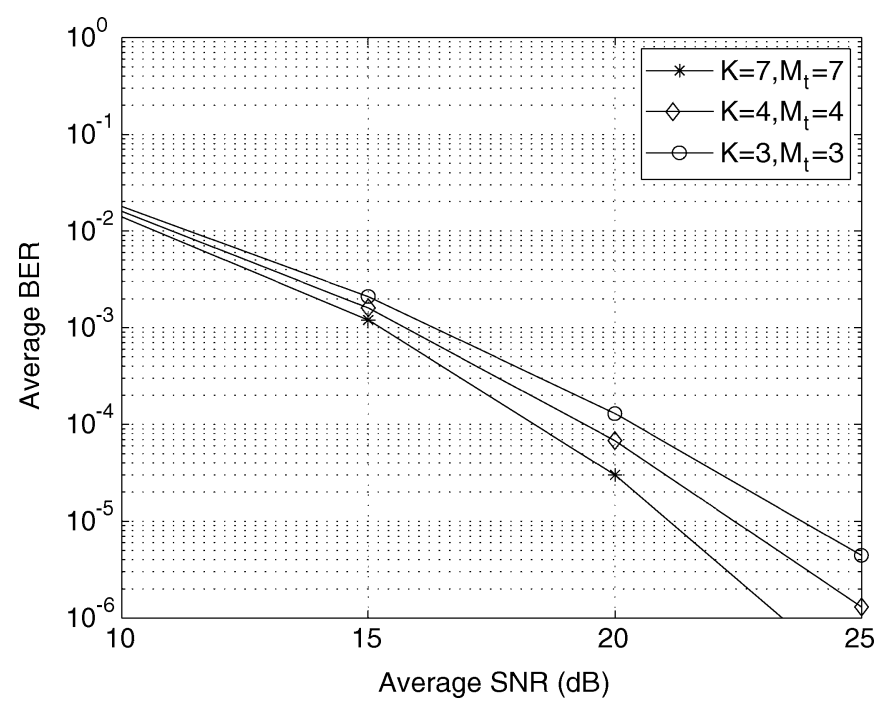

Fig. 6. Average BER performance for multiuser schemes with various number of users and BTS antennas.

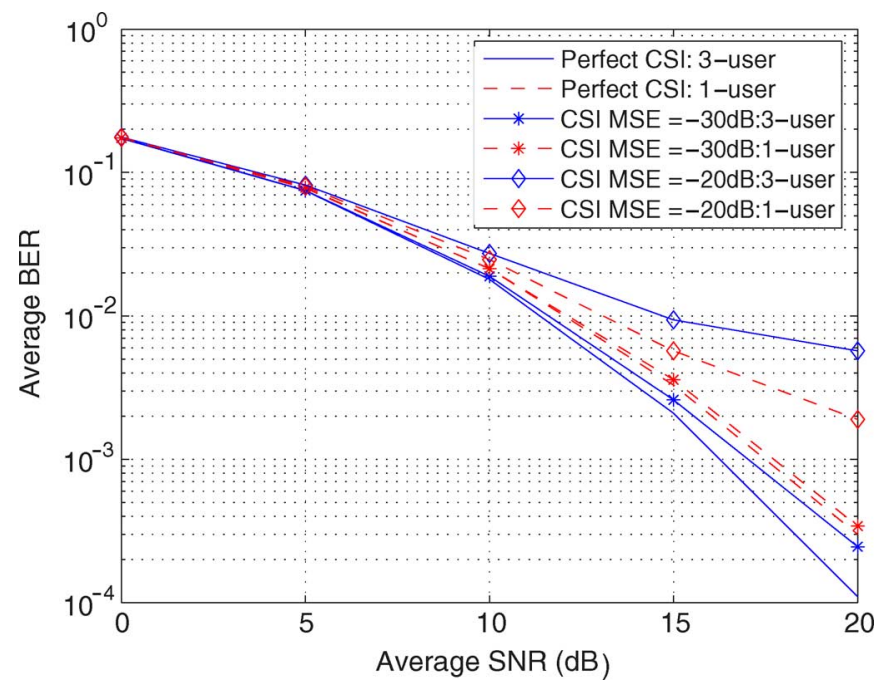

Fig. 7. Performance of a three-user scheme with imperfect CSI (three BTS antennas).

BTS antennas is compared with that of a single-user system with two BTS antennas.

\section{CONCLUSION}

In this paper, we have considered the CCI and ISI suppression for multiuser downlink transmission in frequency-selective fading channels. A single-carrier frequency-domain multiuser transmit beamforming and pre-equalization technique was proposed. The optimal beamforming and pre-equalization vectors were derived by canceling the CCI and minimizing the MSE for each individual user. Our results demonstrated that, in frequency-selective fading channels, the multiuser scheme with $K$ users and $M_{t}$ BTS antennas could offer $K$ times improvement in system throughput while providing improved BER performance over a single-user scheme with $M_{t}-K+1$ BTS antennas.

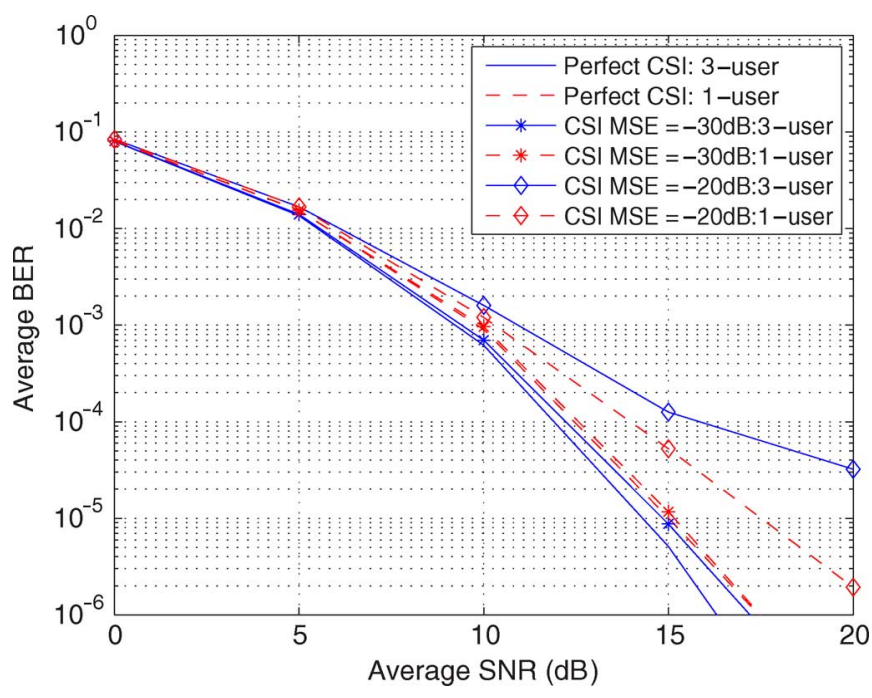

Fig. 8. Performance of a three-user system with imperfect CSI (four BTS antennas).

\section{APPENDIX I \\ CALCUlation OF $\rho_{A_{n}, A_{m}}$}

To obtain $\rho_{A_{n}, A_{m}}$ (30), the key is to calculate:

$$
\mathbb{E}\left[A_{n} A_{m}\right]=\mathbb{E}\left[\sum_{p=1}^{M_{t}} \sum_{q=1}^{M_{t}}\left|H_{p, n}\right|^{2}\left|H_{q, m}\right|^{2}\right] .
$$

As in Section II, the time-domain channel coefficients $h_{p, l}$ are modeled to be zero-mean and complex Gaussian random variables with variance $\sigma_{l}^{2}$. The power of channel $\sum_{l=0}^{L} \sigma_{l}^{2}=1$. The real and imaginary parts of $h_{p, l}$ are independent zero-mean Gaussian random variables with variance $\sigma_{l}^{2} / 2 . h_{p, l}$ is independent for different $p$ and different $l$. So $H_{p, n}$ and $H_{q, m}$ are uncorrelated for $p \neq q$ but are correlated for $p=q$. If $p \neq q$,

$$
\mathbb{E}\left[\left|H_{p, n}\right|^{2}\left|H_{q, m}\right|^{2}\right]=\mathbb{E}\left[\left|H_{p, n}\right|^{2}\right] \mathbb{E}\left[\left|H_{q, m}\right|^{2}\right]=1 .
$$

For $p=q$, we have

$$
\begin{aligned}
\mathbb{E}\left[\left|H_{p, n}\right|^{2}\left|H_{p, m}\right|^{2}\right]=\mathbb{E} & {\left[\left|\sum_{l=0}^{L} h_{p, l} e^{-j \frac{2 \pi}{N} l n}\right|^{2}\right.} \\
& \left.\times\left|\sum_{i=0}^{L} h_{p, i} e^{-j \frac{2 \pi}{N} i m}\right|^{2}\right] \\
=1 & +\rho_{1}^{2}(n-m)+\rho_{2}^{2}(n-m)
\end{aligned}
$$

where

$$
\begin{aligned}
& \rho_{1}(n-m)=\sum_{l=0}^{L} \sigma_{l}^{2} \cos \left(\frac{2 \pi(n-m) l}{N}\right) \\
& \rho_{2}(n-m)=\sum_{l=0}^{L} \sigma_{l}^{2} \sin \left(\frac{2 \pi(n-m) l}{N}\right) .
\end{aligned}
$$


So the correlation between $A_{n}$ and $A_{m}$ is

$$
\begin{aligned}
\mathbb{E}\left[A_{n} A_{m}\right] & =M_{t}^{2}-M_{t}+M_{t}\left(1+\rho_{1}^{2}(n-m)+\rho_{2}^{2}(n-m)\right) \\
& =M_{t}^{2}+M_{t}\left(\rho_{1}^{2}(n-m)+\rho_{2}^{2}(n-m)\right) .
\end{aligned}
$$

After simple derivatives, the correlation coefficient is

$$
\rho_{A_{n}, A_{m}}=\rho_{1}^{2}(n-m)+\rho_{2}^{2}(n-m) .
$$

\section{APPENDIX II}

Calculation of Variance of $B_{n}$

Given $\mathbf{H}_{n}^{(2)}$, the variance of $B_{n}$ in (33) is

$$
\sigma_{B_{n}}^{2}\left(\mathbf{H}_{n}^{(2)}\right)=\mathbb{E}\left[B_{n}^{2} / \mathbf{H}_{n}^{(2)}\right]-M_{t}^{2}
$$

We have

$$
\begin{aligned}
\mathbb{E}\left[B_{n}^{2} / \mathbf{H}_{n}^{(2)}\right] & \\
= & \mathbb{E}\left[\left(\sum_{p=1}^{M_{t}+1}\left|H_{p, n}^{(1)}\right|^{2}\right.\right. \\
= & \left.\left.-\frac{1}{\left.\left\|\mathbf{H}_{n}^{(2)}\right\|\right|^{2}}\left|\sum_{p=1}^{M_{t}+1} H_{p, n}^{(1) *} H_{p, n}^{(2)}\right|^{2}\right)^{2} / \mathbf{H}_{n}^{(2)}\right] \\
+ & \frac{\mathbb{E}\left[\left.\left|\sum_{p=1}^{M_{t}+1} \sum_{q=1}^{M_{t}+1}\right| H_{p, n}^{(1)}\right|^{2}\left|H_{q, n}^{(1)}\right|^{2}\right]}{\|\left.\mathbf{H}_{p, n}^{(1)} H_{p, n}^{(2)}\right|^{4}} \|^{4} \\
& -\frac{2 \mathbb{E}\left[\sum_{p=1}^{M_{t}+1}\left|H_{p, n}^{(1)}\right|^{2}\left|\sum_{p=1}^{M_{t}+1} H_{p, n}^{(1)^{*}} H_{p, n}^{(2)}\right|^{2}\right]}{\left\|\mathbf{H}_{n}^{(2)}\right\|^{2}} .
\end{aligned}
$$

For same $k$ and $p, H_{p, n}^{(k)}$ and $H_{p, m}^{(k)}$ are correlated. The first term on the right-hand side of (51) is $\left(M_{t}+1\right)^{2}+\left(M_{t}+1\right)$. The second term on the right-hand side of (51) is 2 , while the third term on the right-hand side of (51) is $2\left(M_{t}+2\right)$. Thus, we have

$$
\mathbb{E}\left[B_{n}^{2} / \mathbf{H}_{n}^{(2)}\right]=M_{t}^{2}+M_{t}
$$

and

$$
\sigma_{B_{n}}^{2}\left(\mathbf{H}_{n}^{(2)}\right)=M_{t}^{2}
$$

The correlation $\mathbb{E}\left[B_{n} B_{m} / \mathbf{H}_{n}^{(2)}, \mathbf{H}_{m}^{(2)}\right]$ is calculated through

$$
\begin{aligned}
\mathbb{E}[ & \left.B_{n} B_{m} / \mathbf{H}_{n}^{(2)}, \mathbf{H}_{m}^{(2)}\right] \\
= & {\left[\left\langle\sum_{p=1}^{M_{t}+1}\left|H_{p, n}^{(1)}\right|^{2}-\frac{1}{\left\|\mathbf{H}_{n}^{(2)}\right\|^{2}}\left|\sum_{p=1}^{M_{t}+1} H_{p, n}^{(1) *} H_{p, n}^{(2)}\right|^{2}\right)\right.} \\
& \times\left[\sum_{p=1}^{M_{t}+1}\left|H_{p, m}^{(1)}\right|^{2}-\frac{1}{\left\|\mathbf{H}_{m}^{(2)}\right\|^{2}}\left|\sum_{p=1}^{M_{t}+1} H_{p, m}^{(1)^{*}} H_{p, m}^{(2)}\right|^{2}\right) \\
= & {\left[\sum_{p=1}^{M_{t}+1} \sum_{q=1}^{M_{t}+1}\left|H_{p, n}^{(1)}\right|^{2}\left|H_{q, m}^{(1)}\right|^{2}\right] } \\
+ & \frac{\mathbb{E}\left[\left|\sum_{p=1}^{M_{t}+1} H_{p, m}^{(1) *} H_{p, m}^{(2)}\right|^{2}\left|\sum_{q=1}^{M_{t}+1} H_{q, m}^{(1)^{*}} H_{q, m}^{(2)}\right|^{2}\right]}{\left\|\mathbf{H}_{n}^{(2)}\right\|^{2}\left\|\mathbf{H}_{m}^{(2)}\right\|^{2}} \\
- & \frac{\mathbb{E}\left[\sum_{p=1}^{M_{t}+1}\left|H_{p, n}^{(1)}\right|^{2}\left|\sum_{q=1}^{M_{t}+1} H_{q, m}^{(1)^{*}} H_{q, m}^{(2)}\right|^{2}\right]}{\left\|\mathbf{H}_{m}^{(2)}\right\|^{2}} \\
- & \frac{\mathbb{E}\left[\sum_{q=1}^{M_{t}+1}\left|H_{q, m}^{(1)}\right|^{2}\left|\sum_{p=1}^{M_{t}+1} H_{p, n}^{(1) *} H_{p, n}^{(2)}\right|^{2}\right]}{\left\|\mathbf{H}_{n}^{(2)}\right\|^{2}}
\end{aligned}
$$

The first term on the right-hand side of (54) is $\left(M_{t}+1\right)^{2}+$ $\left(M_{t}+1\right)\left(\rho_{1}^{2}(n-m)+\rho_{2}^{2}(n-m)\right)$. The second term on the right-hand side of (54) is

$$
1+\left(\rho_{1}^{2}(n-m)+\rho_{2}^{2}(n-m)\right) \frac{\left|\mathbf{H}_{n}^{(2)^{H}} \mathbf{H}_{m}^{(2)}\right|^{2}}{\mid \mathbf{H}_{n}^{(2)}\left\|^{2}\right\| \mathbf{H}_{m}^{(2)} \|^{2}} .
$$

The third and forth term are equal and given by

$$
M_{t}+1+\rho_{1}^{2}(n-m)+\rho_{2}^{2}(n-m) .
$$

Thus, given $\mathbf{H}_{n}^{(2)}$ and $\mathbf{H}_{m}^{(2)}$, the correlation between $B_{n}$ and $B_{m}$ is

$$
\begin{aligned}
\mathbb{E}\left[B_{n} B_{m} / \mathbf{H}_{n}^{(2)},\right. & \left.\mathbf{H}_{m}^{(2)}\right]=M_{t}^{2}+\left(\rho_{1}^{2}(n-m)+\rho_{2}^{2}(n-m)\right) \\
& \times\left(M_{t}-1+\frac{\left|\mathbf{H}_{n}^{(2)}{ }^{H} \mathbf{H}_{m}^{(2)}\right|^{2}}{\left\|\mathbf{H}_{n}^{(2)}\right\|^{2}\left\|\mathbf{H}_{m}^{(2)}\right\|^{2}}\right)
\end{aligned}
$$

\section{REFERENCES}

[1] L. Guo and Y. F. Huang, "A multiuser multi-antenna system for downlink transmission in frequency-selective fading channels," presented at the Proc. IEEE Int. Workshop Signal Processing Advances in Wireless Communications, New York, Jun. 5-8, 2005. 
[2] E. Telatar, "Capacity of multi-antenna gaussian channels," Euro. Trans. Commun., vol. 10, no. 6, pp. 585-595, Nov.-Dec. 1999.

[3] G. J. Foschini and M. J. Gans, "On limits of wireless communications in a fading environment when using multiple antennas," Wireless Personal Commun., vol. 6, no. 3, pp. 311-335, Mar. 1998.

[4] H. Weingarten, Y. Steinberg, and S. Shamai, "The capacity region of the Gaussian MIMO broadcast channel," in Proc. IEEE Int. Symp. Inf. Theory (ISIT), Jun. 27-Jul. 2, 2004, p. 174.

[5] P. Viswanath and D. N. C. Tse, "Sum capacity of the multiple antenna Gaussian broadcast channel," in Proc. IEEE Int. Symp. Information Theory, Palais de Beaulieu, Lausanne, Switzerland, Jun. 31-Jul. 5, 2002 , p. 497.

[6] W. Yu and J. M. Cioffi, "Sum capacity of Gaussian vector broadcast channels," IEEE Trans. Inf. Theory, vol. 50, no. 9, pp. 1875-1892, Sep. 2004.

[7] G. Caire and S. Shamai, "On the achievable throughput of a multiantenna Gaussian broadcast channel," IEEE Trans. Inf. Theory, vol. 43, pp. 1691-1706, Jul. 2003.

[8] S. Vishwanath, N. Jindal, and A. Goldsmith, "Duality, achievable rates and sum capacity of Gaussian MIMO broadcast channels," IEEE Trans. Inf. Theory, vol. 49, pp. 2658-2668, Aug. 2003.

[9] M. Joham, W. Utschick, and J. A. Nossek, "Linear transmit processing in MIMO communications systems," IEEE Trans. Signal Process., vol. 53, no. 8, pp. 2700-2712, Aug. 2005.

[10] M. Schubert and H. Boche, "Solution of the multiuser downlink beamforming problem with individual SINR constraints," IEEE Trans. Veh. Technol., vol. 53, no. 1, pp. 18-28, Jan. 2004.

[11] Q. H. Spencer, A. L. Swindlehurst, and M. Haardt, "Zero-forcing methods for downlink spatial multiplexing in multi-user MIMO channels," IEEE Trans. Signal Process., vol. 52, no. 2, pp. 461-471, Feb. 2004.

[12] K. K. Wong, R. D. Murch, and K. B. Letaief, "Performance enhancement of multiuser MIMO wireless communication systems," IEEE Trans. Commun., vol. 50, no. 12, pp. 1960-1970, Dec. 2002.

[13] R. Chen, J. G. Andrews, and R. W. Heath, Jr., "Multiuser spacetime block coded MIMO systems with downlink precoding," in Proc. 2004 IEEE Int. Conf. Communications, Paris, France, Jun. 2004, vol. 5, pp. 2689-2693.

[14] L. Q. Hoo, B. Halder, J. Tellado, and J. M. Cioffi, "Multiuser transmit optimization for multicarrier broadcast channels: Asymptotic FDMA capacity region and algorithm," IEEE Trans. Commun., vol. 52, no. 6, pp. 922-930, Jun. 2004

[15] Y. W. Cheong, C. Y. Tsui, R. S. Cheng, and K. B. Letaief, "A real-time subcarrier allocation scheme for multiple access downlink OFDM transmission," in Proc. IEEE Vehicular Technology Conf., Amesterdam, The Netherland, Sep. 1999, vol. 2, pp. 1124-1128.

[16] L. M. C. Hoo and J. M. Coiffi, "FDMA-based multiuser transmit optimization for broadcast channels," in Proc. IEEE Wireless Communication Networking Conf., Chicago, IL, Sep. 2000, vol. 2, pp. 597-602.

[17] W. Rhee and J. M. Coiffi, "Increase in capacity of multiuser OFDM system using dynamic subchannel allocation," in Proc. IEEE Vehicular Technology Conf., Tokyo, Japan, May 2000, vol. 2, pp. 1085-1089.

[18] J. Duplicy, J. Louveaux, and L. Vandendorpe, "Interference-free multiuser MIMO-OFDM," in Proc. IEEE Int. Conf. Acoustics, Speech, Signal Processing, 2005, Mar. 18-23, 2005, vol. III, pp. 1149-1152.

[19] D. Falconer, S. L. Ariyavisitakul, A. Benyamin-Seeyar, and B. Eidson, "Frequency domain equalization for single-carrier broadband wireless systems," IEEE Commun. Mag., vol. 40, no. 4, pp. 58-66, Apr. 2002.

[20] N. Al-Dhahir, "Single-carrier frequency-domain equalization for space-time block-coded transmissions over frequency-selective fading channels," IEEE Commun. Lett., vol. 5, no. 7, pp. 304-306, Jul. 2001.

[21] M. V. Clark, "Adaptive frequency-domain equalization and diversity combining for broadband wireless communications," IEEE J. Sel. Areas Commun., vol. 16, no. 8, pp. 1385-1395, Oct. 1998.

[22] L. Choi and R. D. Murch, "A transmit MIMO scheme with frequency-domain pre-equalization for wireless frequency-selective channels," IEEE Trans. Wireless Commun., vol. 3, no. 3, pp. 929-938, May 2004.
[23] S. Budisin, "Golay complementary sequences are superior to PN sequences," in Proc. 1992 IEEE Int. Conf. Systems Engineering, Sep. 17-19, 1992, pp. 101-104.

[24] B. J. Seberry, B. J. Wysocki, and T. Wysocki, "Olay sequences for DS-CDMA applications," in Proc. 6th Int. Symp. DSP for Communication Systems, Sydney-Manly, Jan. 2002, pp. 103-108.

[25] R. Stelle and L. Hanzo, Mobile Radio Communications, 2nd ed. New York: Wiley, 1999.

[26] J. H. Winters, J. Salz, and R. D. Gitlin, "The impact of antenna diversity on the capacity of wireless communication systems," IEEE Trans. Commun., vol. 42, no. 2/3/4, pp. 1740-1751, Feb./March/Apr. 1994.

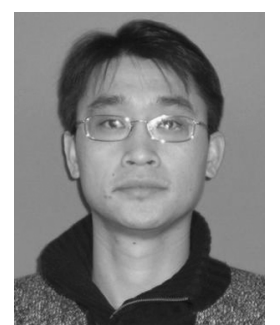

Li Guo received the B. Eng. degree in radio communication engineering from Beijing University of Posts and Telecommunications, Beijing, China, in 1998 and the M.S.E.E. degree and the Ph.D. degree in electrical engineering from the University of Notre Dame, IN, in 2004 and 2007, respectively.

$\mathrm{He}$ was a Senior Systems Engineer at Navin Networks, Inc., Richardson, TX, during 2006-2007. Since 2007, he has been with Cisco Systems, Inc., Richarson, TX, where he conducts research on multiantenna technologies and broadband wireless technologies. His research interests include adaptive signal processing, statistical estimation and detection theory, multiuser and multiantenna system, and broadband wireless access.

Mr. Guo placed second in the final student paper contest at the 39th Asilomar Conference on Signals, Systems, and Computers, November 2005.

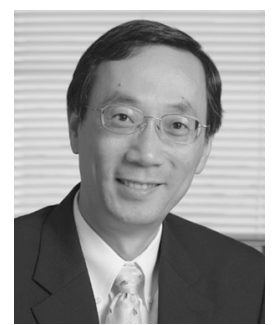

Yih-Fang Huang (F'95) received the B.S.E.E. degree from National Taiwan University, Taiwan, R.O.C., in 1976, the M.S.E.E. degree from the University of Notre Dame, IN, in 1980, and the Ph.D. degree in electrical engineering from Princeton University, Princeton, NJ, in 1982.

Since 1982, he has been on the faculty at the University of Notre Dame, where he is currently Professor of Electrical Engineering. He served as Chair of the Department of Electrical Engineering at Notre Dame from 1998 to 2006. In Spring 1993, he was Toshiba Visiting Professor at Waseda University, Tokyo, Japan, in the Department of Electrical Engineering. In summer 2007, he was Visiting Professor at the Technical University of Munich, Germany. His research interests focus on statistical communications and adaptive signal processing. Collaborating with his students and colleagues, he has developed set-membership filtering (SMF) and derived a group of adaptive algorithms known as optimal bounding ellipsoids (OBEs). His recent interests are in applications of SMF to multiple-access and wireless communication systems, particularly on issues of interference mitigation.

Dr. Huang was the area editor on mathematics for Circuits and Filters Handbook (CRC Press/IEEE Press, 1995) and Signal Processing for the Electrical Engineering Handbook (Elsevier/Academic Press, 2005). He has been on the Editorial Board for the Journal of Franklin Institute. He was Associate Editor for the IEEE TRANSACTIONS ON CIRCUITS AND SYSTEMS from 1989 to 1991 and Associate Editor for its EXPRESS LETTERS in 1992-1993. He was Vice-President for the IEEE Circuits and Systems Society in 1997-1998 and was a Distinguished Lecturer for the same society in 2000-2001. He was awarded the University of Notre Dame's Presidential Award in 2003, and the Golden Jubilee Medal of the IEEE Circuits and Systems Society in 1999. In Fall 2007, he was a recipient of the Fulbright-Nokia scholarship, visiting Helsinki University of Technology. 